\title{
Evolutionary Systems. A Manifesto ${ }^{\dagger}$
}

\author{
Rainer E. Zimmermann 1,2,3,*,‡, Wolfgang Hofkirchner ${ }^{4}$, José M. Díaz Nafría ${ }^{5}$, Annette Grathoff ${ }^{6}$, \\ Tomáš Sigmund ${ }^{7}$ and Xiaomeng Zhang 8,9 \\ 1 Lehrgebiet Philosophie, FK 13, Hochschule München, 80636 München, Germany; \\ rainer.zimmermann@hm.edu \\ 2 Clare Hall, Cambridge CB3 9AL, UK \\ 3 Institut für Design Science, 80797 München, Germany \\ 4 Bertalanffy Center for the Study of Systems Science, 1040 Vienna, Austria; wolfgang.hofkirchner@bcsss.org \\ 5 Didáctica General, Específicas y Teoría de la Educación, Universidad de León, 24071 León, Spain; \\ jdian@unileon.es \\ 6 Institut für Design Science, 80797 München, Germany; a.grathoff@uni-oldenburg.de \\ 7 Systems Analysis Department, Faculty Informatics and Statistics, University of Economics, 13067 \\ Prague, Czech Republic; tomas.sigmund@gmail.com \\ 8 Liberal Arts Department, Renmin University, 100872 Bejing, China; utopia_1990@163.com \\ 9 Ernst-Bloch-Zentrum, Ludwigshafen, Germany \\ * Correspondence: pd00108@mail.lrz-muenchen.de \\ + Presented at the IS4SI 2017 Summit DIGITALISATION FOR A SUSTAINABLE SOCIETY, Gothenburg, \\ Sweden, 12-16 June 2017. \\ $\ddagger$ This is a provisional version of the manifesto that is still under consideration and up to discussion \\ Published: 11 September 2017
}

After roughly 35 years of development in the theories of self-organization and related variants (chaos, self-organized criticality, and so forth), it is somewhat of a surprise that physics proper has not yet sufficiently found its entry into the ongoing quest for a precise concept of information. Already as early as in the sixties of the last century, Fredkin and Zuse visualized the universe altogether as a digital computer, a line of argument that Wolfram has followed more recently. Not to speak of the even more recent theories on quantum information that emerged during the nineties and tended from the beginning on to coupling generically to theories of quantum gravity. As Deutsch $([3], 93,100)$ has formulated: “[...] [b]its, Boolean variables, and classical computation are all emergent or approximate properties of qubits, manifested mainly when they undergo de-coherence $[\ldots]$. The world is made of qubits [...]. What we perceive to some degree of approximation as a world of single-valued variables is actually part of a larger reality in which the full answer to a yes-no question is not just yes or no, nor even both yes and no in parallel, but a quantum-observable-something that can be represented as a large Hermitian matrix". In fact, we would rather tend to add energy-mass ( = matter) to this as a second register, because, if visualizing the universe as a quantum computer, this means that a computer consists of both hardware and software, respectively. Energy (or matter as to that) stands for the former, information for the latter. This viewpoint goes actually back as far as to John Wheeler in 1977 whose perspective led at the time to the famous "it-from-bit" thesis proposing that "the universe be fundamentally an information processing system from which the appearance of matter emerges at a higher level of reality". (Davies $[2], 10)$ In fact, very much in the sense of Deutsch [3], both Zizzi [16] and Lloyd ([6,7]) generalized this thesis by replacing it in terms of a new "it-from-qubit" thesis. It is in particular Lloyd who developed the cosmological implications in detail when presenting his work in 2006 and 2010. For him, the big bang was also a bit bang. Hence, within our picture, it is ultimately both matter and information that show up as two different aspects of the same underlying primordial structure. It is especially in loop quantum gravity that these features are most prominent, the adequate modelling language being that of topos theory. In the meantime, recent work on the concept of quantum de-coherence (Schlosshauer [8]) as well as on its origin in gravity itself (Zych, Pikovksi, Costa, Brukner [17]) has clarified this viewpoint even more. From this development in physics and its 
philosophical conceptualization we can draw a number of relevant conclusions which are listed in the following manifesto.

Proposition 1. If a theory of cosmology must, as Smolin $([9], 291)$ puts forward, in order to be self-consistent, be a theory of the self-organization of the universe, then the very concept of organization entails a concept of meaningful information (in the sense of Floridi's project, though possibly not on his line of argument) from the outset on an equal footing with the concept of energy-mass. Hence, similar to the latter, information is already always present in the fundamental physics which is concerned with the fabric of the world and its physical grounding. Both energy-mass (matter) and information are thus physical. Hence, they are categories of the observable world. Nevertheless, they show up as two different aspects of the same underlying primordial structure which in loop quantum gravity can be visualized in terms of the spin network consisting of loops that co-operate in order to form a framework of hexagonal structures. Obviously, the afore-mentioned primordial structure is to the observable world almost what in terms of classical meta-physics substance is to attribute.

Proposition 2. If we follow the argument of Seth Lloyd ([6], 26, [7]), then the afore-mentioned information is in particular meaningful from the beginning on: "If you adopt Wittgenstein's perspective that the meaning of a piece of information is to be found in the action this information provokes, the meaning of a computer program written in a particular computer language is to be found in the actions the computer performs as it interprets that program." This has the interesting consequence that a loop in the above sense fulfils what Stuart Kauffman calls the criteria for autonomous agents. Hence, as the spin network essentially represents the last layer of the observable world (displaying quantum forms of the observable categories of space, time, energy, matter, entropy, and structure) and is constituted by co-operating fundamental agents, this entails a concept of proto-subjectivity which is primarily based on self-organizing systems. Note that the unified ground of the observable world, expressed in terms of these categories, constitutes thus the worldly subject proper (hypokeimenon) different from substance (ousía) as known in the Aristotelian terminology of meta-physics. Consequently, human subjectivity shows up in the end as a superposition of such proto-subjective sub-systems approaching a (so far) maximal complexity. At the same time, more in metaphysical rather than simply physical terms, the origin of information shows up within this context not simply as difference (as opposed to identity) alone, but instead as difference of identity and difference and its sublation in what Schelling calls indifference.

Remark 1. Note the loop of self-reference which is always intrinsic to all reflexive activities performed by human beings that are products of the world they would like to model: Hence, only in terms of given social context is it thus possible to perform a modelling procedure at all. This is why we have two immanent aspects here deriving from classical approaches of philosophy: (a) the model itself, scientific or otherwise, is always grounded within a social context (a prominent line of argument taken by theories in the Marxist tradition up to the Frankfort school); (b) we have always to take the ontological difference into account, between modality (the world as it is observed) and reality (the world as it really is independent of observation) - also referring to a long philosophical tradition up to phenomenology and fundamental ontology in the sense of the early Heidegger. Indeed, joint work presently under way (Zimmermann, Zhang [15]) indicates the relevance of connecting these aspects with the emergence of reflexive contrast within the human consciousness itself, a concept that derives from the line of argument given by Ernst Bloch in terms of what he calls "the darkness of the lived moment". (Zimmermann [13]).

Proposition 3. Hence, the universe is meaningful from the beginning on as meaning emerges alongside with energy-mass (matter) and information at the big bang (whatever this ground state may be according to recent insight from quantum gravity). Subsequently however, the evolution of organization entails the evolution of (organized) systems as well as the evolution of meaning such that this evolution is characterized by emergent steps of its developmental complexity. Because it is the evolution of the forthcoming levels of complexity (following the fourth law of thermodynamics according to Stuart Kauffman) that demonstrates the conceptual multi-perspectivity of univocity, analogy, and equivocity, respectively, on each such level, there is no reason to associate with the latter triad an epistemic difficulty which after Capurro is used to be called "trilemma". (Capurro et al.,[1], 9) Instead, this triad simply unfolds the local perspective of conceptualization with respect to that level of complexity which is topical for a given stage of development. 
Proposition 4. The complete picture can thus be summarized as follows:

\begin{tabular}{ccc}
\hline & Energy - Mass = Matter & Entropy - Structure = Information \\
\hline potential form & state of a system & possible organization \\
actual form & state of a system/of mass & state of organization (structure) \\
local form & balance equation & balance equation \\
global form & conservation law & positive entropy balance \\
total quantity & $\mathrm{E}=0$ (incl. matter) & $\mathrm{S}=0$ (incl. structure)? \\
\hline
\end{tabular}

In other words: Both matter and information can be discussed in parallel, but they cannot be identified, because the latter is prescribing how to organize what the former provides as the necessary capacity of work. Hence, energy can be both potential or actual, respectively, while mass is actualized energy. On the other hand, entropy is potential information, while structure is actualized information and can serve as memory storage of its own evolution. (Zimmermann [14]) Whether it is useful to associate entropy with what Stonier ([10]) calls "kinetic information", and the rest with what he calls "structural information" is presently under debate. But it is clearly worthwhile to look for a possible reconciliation of the concepts involved on this line of arguments, especially with a view to biological insight.

Proposition 5. The above-mentioned propositions entail the following definitions which are compatible with what has been introduced so far (Zimmermann [11]).

Definition 1. We call system a network of interacting agents producing a space with a well-defined boundary that is open in the sense of thermodynamics.

Remark 2: Note that the definition of an agent entails the aspect of self-organization. Interaction of agents is visualized as competition within co-operation in the sense of Eigen and Schuster and is thus accessible by game theory. (S. Kauffman [5]) This essentially relational aspect is discussed in particular in Hofkirchner [4].

Definition 2. We call ground of a system from what, by what, and because of what a system actually emerges.

Corollary 1. Hence, ground is to system what non-being is to being. (Note that existence is thus visualized as (permanent) transformation of non-being into being.)

Corollary 2. The ground of the ground (or: primordial ground) is the non-being of non-being (i.e. the condition of non-being) and is called nothingness.

Proposition 6. To be more precise, it is energy-mass on the one hand and entropy-structure on the other that show up as two different aspects (attributes) of the primordial ground (substance) mediated by the unified subject of the world as expressed in terms of loops organized in spin network hexagons (in the sense of Roger Penrose). Substance can be visualized here as being represented by a primordial state of quantum coherence within which space, time, and matter as to that (and information) are in fact absent in the categorial sense. The objective of a physical theory of everything is the accessing of the unified subject. But the accessing of substance is the objective of meta-physics. (Zimmermann [12]) Although there is thus an algorithmic connotation to all of this referring to the characteristics of evolutionary systems in the first place, the interpretation of meaningful information has also to rely on hermeneutic techniques in order to complete this aforementioned accessing. Substance is the procedural space onto which the subject is grounded. Substance is to the observable world what software (procedural space) is to what we can see on the computer screen (the space of actualized procedures). The difference is only that human beings are intrinsic observers and thus part of the screen. Also, if we think of fashionable three-dimensional printers, their software is nothing without appropriate hardware. 


\section{References}

1. Capurro, R.; Fleissner, P.; Hofkirchner, W. Is a Unified Theory of Information Feasible? A Trialogue (e-mail discussion). In The Quest for a Unified Theory of Information; Hofkirchner, W., Ed.; Gordon \& Breach: Amsterdam, The Netherlands, 1999; pp. 9-30.

2. Davies, P.C.W. John Archibald Wheeler and the clash of ideas. In Science and Ultimate Reality. Quantum Theory, Cosmology, and Complexity; Barrow, J.D., Davies, P.C.W., Charles, H., Jr., Eds.; Cambridge University Press: Cambridge, UK, 2004; pp. 3-23.

3. Deutsch, D. It from qubit. In Science \& Ultimate Reality; Barrow, J., Davies, P., Harper, C., Eds.; Cambridge University Press: Cambridge, UK, 2004; pp. 90-102.

4. Hofkirchner, W. Emergent Information; World Scientific: Singapore, 2013.

5. Kauffman, S. Investigations; Oxford University Press: Oxford, UK, 2000.

6. Lloyd, S. Programming the Universe; Vintage (Random House): New York, NY, USA, 2007.

7. Lloyd, S. The computational universe. In Information and the Nature of Reality. From Physics to Metaphysics; Davies, P.C.W., Gregersen, N.H., Eds.; Cambridge University Press: Cambridge, UK, 2010; pp. 92-103.

8. Schlosshauer, M.A. Decoherence and the Quantum-to-Classical Transition; Springer: Berlin/Heidelberg, Germany, 2007.

9. Smolin, L. The Life of the Cosmos; Oxford University Press: Oxford, UK, 1997.

10. Stonier, T. Information und Die Innere Struktur des Universums; Springer: Berlin/Heidelberg, Germany, 1991.

11. Zimmermann, R.E. Nothing as Ground and Nothing but Ground. Schelling's Philosophy of Nature Revisited; Xenomoi: Berlin, Germany, 2014.

12. Zimmermann, R.E. Metaphysics of Emergence. Part I: On the Foundations of Systems; Xenomoi: Berlin, Germany, 2015.

13. Zimmermann, R.E. (Ed.) Ernst Bloch: Das Prinzip Hoffnung. In Klassiker Auslegen; de Gruyter: Berlin, Germany; Boston, MA, USA, 2017; Volume 56.

14. Zimmermann, R.E. Matter and Information as Attributes of Substance. Eur. Phys. J. Spec. Top. 2017, 226, 177-180.

15. Zimmermann, R.E.; Zhang, X. Sayable and Unsayable Within Lived Immediacy. 2017, in press for Bloch Yearbook, Ludwigshafen.

16. Zizzi, P.A. Holography, Quantum Geometry, and Quantum Information Theory. Entropy 2000, 2, 39-69.

17. Zych, M.; Pikovski, I.; Costa, F.; Brukner, Č. General relativistic effects in quantum interference of “clocks". J. Phys. 2016, 723, 012044.

(C) 2017 by the authors. Licensee MDPI, Basel, Switzerland. This article is an open access article distributed under the terms and conditions of the Creative Commons Attribution (CC BY) license (http://creativecommons.org/licenses/by/4.0/). 